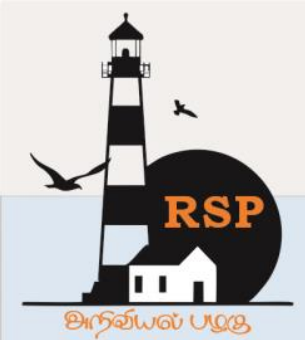

INTERNATIONAL RESEARCH JOURNAL ON ADVANCED SCIENCE HUB

\title{
Future of EEG Based Hybrid Visual Brain Computer Interface Systems in Rehabilitation of People with Neurological Disorders
}

\section{Dr. Deepak Kapgate}

Assistant Professor Nagpur University, Nagpur, India.

deepakkapgate32@gmail.com ${ }^{1}$

\section{Abstract}

\begin{abstract}
Brain-computer interfaces (BCIs) are technologies that make it possible for humans to control external devices merely using their cortical potentials rather than normal output pathways such as muscles or peripheral nerves. BCIs present a hope towards restoration of independence for people affected by neurological disorders or disable individuals. Hybrid visual BCI (V-BCI) i.e. BCIs those are using two or more types of visual evoked potential for its operation, are providing promising results than other all $B C I$ systems types. Over past two decades research and development in hybrid V-BCI systems have grown tremendously. Recently lot of efforts has been placed to make laboratory validated hybrid V-BCI systems to work in real life applications for disables. In this paper we argue on possible futuristic applications of hybrid V-BCI systems and its clinical relevance. We will present existing restrictions of hybrid $V$-BCI technology and its futuristic expectations.
\end{abstract}

Keywords: V-BCI systems, EEG, Neurological Disorders ,etc

\section{Introduction}

Brain-Computer Interfaces (BCI) created as a tool for assisting the disabled as well as healthy humans of society by mapping of individual intentions onto directive commands in professional and daily tasks [1]. Performing tasks such as controlling external devices by measuring and analyzing cortical activity is possible in electroencephalogram (EEG) based brain-computer interface (BCI). Compared to other neuroimaging methods, electroencephalography (EEG) is widely used because of its high temporal resolution, high portability, few risks to users and relative low cost [2]. BCIs that utilize visual evoked potentials (VEPs) for its operation attain popularity in last few years than other BCI types because of their higher communication rate, ease of usability and less/negligible training time [3]. Most prominent BCIs based on VEPs are P300 based BCI and SSVEP based BCI. However conventional BCI have several limitations and "Ideal BCI System" properties are difficult to obtain using conventional BCI. Hence to overcome these limitations a novel approach is proposed by combining conventional BCI system with other interface systems called hybrid BCI [4]. A study of combination of conventional BCI with other physiological/external signals is out of the scope of this paper, so hereafter hybrid BCI term refers to the BCI systems where one or more neurological signals are combined [5]. Various hybrid systems combine two or more cortical potentials based BCI's either in sequential or simultaneous ways. These hybrid systems overcome limitations of conventional BCI systems to some extent. Such as hybrid systems are having higher communication rate, accuracy, robustness and response time as compared to conventional single cortical potential based BCI systems [6]. Although much promising results are achieved by Hybrid BCIs in some research studies, still hybrid BCIs are in naïve stage of development. In very short period, BCI community has made 
considerable progress in research in hybrid BCIs with significant diversity in development of hybrid BCIs [7]. Hybrid BCI systems that use visual evoked potentials for their operation hereafter called as hybrid visual BCI are providing promising results than other Hybrid BCI systems types [8]. So, probable future of Hybrid V-BCI systems must be analyzed to work towards systematic development of these systems. Here in this paper we are recognizing the potential applicable areas of hybrid V-BCI systems and advancement of in such domains using Hybrid V-BCI.

\section{Future of Hybrid V-BCI}

Hybrid V-BCI systems have tremendous potential to develop effective and efficient futuristic task oriented BCI applications. Term task oriented defines applications where user's intentions towards performing online task with real time feedback system and include:

1) BCI based primary interface where users are explicitly performing task using their brain potentials, such as prosthetic movement using modulation of brain potentials.

2) An application where BCI does not provide primary interface but directly supports users performing task. Such as, a system that predicts user's performance while Driving by directly monitoring user's brain signals [9].

In future developers will probably find success in developing task oriented hybrid V-BCIs with possibility of automatic controlling of environmental conditions, where user is intended to perform task. Future hybrid V-BCI systems will turn into prevalent in daily life activities by analyzing cortical potentials for extended time periods with advanced adaptive computing algorithms, artificial intelligence, incorporation of pervasive computing, sensor technologies and brain sensing in multi-aspects [10]. Users wearing brain sensors regularly for specific purposes increase the possibility of hybrid V-BCI systems in "opportunistic" applications. Opportunistic BCI are BCI which do not directly supports user performing task but provide many benefits without any overhead. Let an example of opportunistic BCI as, a pervasive application that automatically adjust local environmental factors (such as dietary suggestions, lightning color, entertainment, music and so on) where user is performing task to enhance or alter user's mental condition or mood [9]. Opportunistic BCI will likely to require collection of brain data in large scale and analyzing data over longer period of time.

\subsection{Direct Control}

Earlier traditional conventional BCI applications were focused towards direct control of external devices or environment by manipulation of cortical activity (i.e. prosthetics devices, wheelchair, computer screen navigation, game control and so on). Hybrid V-BCI proved its significance toward improvement of performance and user conformability over conventional BCI in such applications [11]. In future, there will be likely demand of using hybrid V-BCI in direct control mode in entertainment and quality of life applications. In clinical applicability view, patients with neurological disorders are greatly benefited from use of hybrid V-BCI for consciously control of devices in their surrounding environment and for their own movements. From the entertainment view, ability to control objects directly using their brain would be fascinating to humans, for example telekinesis [12]. There are numerous factors that affect success of direct control.

1) Abilities of brain functioning and its limitations: Although, human brain is capable of various fascinating complex tasks such as motor control, emotion control and so on, it is also apparent that human brain has certain limitations like multilimb control [11]. Let a frictional example where a human is capable of control additional four mechanical limbs together with his own limbs, a concept demonstrated in spider-man movie. Take advantages of such benefits (multilimb control) may require multitasking, which is tedious and difficult to humans.

2) Technical limitations: Non-invasive EEG based V-BCI systems are focused on brain's cortex activity only. Use of brain activities at brain stem and spinal cord is not possible using this technology [13]. This imposes further limitations on applicability of BCI in real life environment.

3) Cortical potentials type: Different types of neural signals signify different brain activities. None of these types of signals fully represent whole brain function. Further, present signal processing algorithms are capable of extract relevant information from little segment of the EEG signal 
[14]. Also brain is dynamic, adaptive and nonstationary.

4) Alternative technologies: In direct control scenario, the main alternative for healthy humans is using their own hands to operate devices). To date, the technologies for directly controlling devices using brain signals provide relatively low bandwidth and low signal-to-noise ratios. As a result, it is a nontrivial problem to enhance control for healthy individuals through the incorporation of brain signals into directcontrol BCIs [14]. For this to occur, the ability to analyze neural signals to add information above and beyond that more easily obtained through other channels (e.g., manual input) will need to be achieved.

\subsection{Indirect Control}

One of the factors that will affect future hybrid V$\mathrm{BCI}$ is inclusion of brain signals in system that could provide information which is not readily accessible from other channels. For example, cortical potentials those are related with human perceptions like comprehension, anger, frustration, attention or error associated signals combination. Indirect control in applications is a control system where user is not engaged directly in task execution. For example, in hybrid V-BCI systems, automatic adjustment of visual stimulus features such as color, frequency, intensity based on strength of cortical potentials evoked in brain in different environmental conditions [15]. In this example application indirectly accesses strength of evoked potentials in human brain and to optimize them further in subsequent task operations, it adjusts external visual stimulus properties, but it will not directly involve in task operations or task control. Mostly success of such applications depends on specificity, robustness and signal detection timeliness. At present there is very restricted applicability of indirect control systems. In future, extensions of hybrid V-BCI systems for indirect controlling of other environmental factors than merely direct task control are needed, to further extend boundaries of Hybrid V-BCI applications in larger context [16].

\subsection{Communications:}

BCI systems have largest impact on communication domain. Previous BCI based communications enables populations of clinical sector such as "locked-in" patients with neurological disorders with slight movement capabilities and small control over devices like prosthetics [17]. These systems have very limited purposes and great benefits to clinical populations rather than healthy populations [20]. Future hybrid V-BCI technologies will make revolutionary advances such as approaching towards communication basis, speech generation and capability to convey meaning among two or more parties [18]. Future of hybrid V-BCI will leverages communications-definite technologies with incorporation of other technologies, like pervasive computing and internet of things [19]. Particularly, hybrid V-BCIs would potentially support to communication domain through three models:

1) Bandwidth among the computer and the human should be increased and bandwidth effectiveness must be enlarged [21].

2) Context information comprehensions must be enhanced or predicted [22].

3) New ideas formation must be supported [23].

\subsection{Brain-Process Modification:}

Futuristic hybrid V-BCI systems may allow capabilities to users to dynamically alter or modify their own brain states or processes. Present technologies like neurofeedback already permit users to regulate their individual brain function to conquer an extra enviable brain state [24 - 27]. Incorporation of analytical approaches and sensor technologies to existing BCI system based neurofeedback can increase its potential benefits. Certain mental disorders like depression, can be treatable by allowing individuals to modulate their own brain states using highly developed neurofeedback system with hybrid V-BCI [28 - 33]. Further unimaginable achievements such as stoppage of neural degradation by ailment or delay age related adaptations can be achieved through allowing user to enter in particular brain state. Current neural stimulation require invasive operations, which can be replaced by non-invasive neural stimulation techniques like magnetic fields, infrared light, direct current, or ultrasound in future [34 - 38] . There could be possibility of putting user in desired brain state using neural stimulation to perform relevant task. However it requires precise determining individual optimal neural goals and strong ethics consideration $[39,40]$.

\subsection{Neurorehabilitation}

In addition to above applications, Hybrid V-BCI system has ability to control prosthetic devices to assist people who have damaged their motor function due to neurological disorders [41]. Improving quality of life of clinical patients and encouraging the neuro- 


\section{www.rspsciencehub.com}

functional recovery using valuable use of damaged brain areas is made possible using neurorehabilitation. Classification of BCI based muscular movement training strategies into two categories is proposed in [42].

1) In first strategy, patients were trained to evoke stronger brain activity in brain with external visual stimulus to organize motor functioning [43]. In EEG based neurorahabilitation, stroke patients skilled to produce particular brain activity patterns such as ERD/ERD signals and BCI is used to collect and analyze these EEG signals to generate control commands and feedback [44].

2) In second strategy, BCI output is used as command signal to devices such as prosthetics to assist movement of patients resulting in improved motor control [45]. This is based on hypothesis that improved motor control using external devices in patients' results in enhanced CNS plasticity [46].

\section{Conclusion}

EEG based hybrid V-BCI demonstrated its abilities to provide motor control capabilities for people with sever motor disabilities. This non-invasive hybrid VBCI systems success will depend on further development in their convenience and ease in daily life use, safety, long term use reliability and applicability in maximum number of tasks. hybrid VBCI systems will be able to induce activity dependent CNS plasticity in patients with neurological disorders using neurorehabilitation. Progress in our understanding of this relation will allow us to predict the amount of the applications of hybrid V-BCI technology in rehabilitation protocols.

\section{References}

1. Adolphs R, The social brain: neural basis of social knowledge, Annu Rev Psychol. 2009; 60: 693-716.

2. Allison B.Z., McFarland D.J., Schalk G., Zheng S.D., Jackson M.M., Wolpaw J.R., Towards an independent brain-computer interface using steady state visual evoked potentials, Clin Neurophysiol, 2008, 119(2):399-408.

3. D. Kapgate et.al. (2020), "An optimized facial stimuli paradigm for hybrid SSVEP+P300 brain computer interface", Cognitive Systems Research, Volume 59, Pages 114-122, DOI: https://doi.org/10.1016/j.cogsys.2019.09.014

4. Yin E et. al.:A speedy hybrid BCI spelling approach combining P300 and SSVEP. IEEE
Volume 02 Issue 06 June 2020

Transaction on Biomed Eng 61(2) pp.:473-483 (2014).

5. Panicker RC et. al.:An asynchronous P300 BCI with SSVEP based control state detection. IEEE Transaction Biomed Eng 58(6): 1781-1788, (2011). 6. Y. Li et. Al.:A Hybrid BCI System Combining P300 and SSVEP and Its Application to Wheelchair Control; IEEE transactions on bio-medical engineering; 60(11); 3156 - 3166 (2013).

7. M. Wang et. al.: A new hybrid BCI paradigm based on P300 and SSVEP, Journal of Neuroscience Methods, 244:16-25 (2015).

8. B. Allison, et. al.: A four-choice hybrid P300/SSVEP BCI for improved accuracy, BrainComputer Interfaces (2014).

9. 96 G. Garipelli, F. Gala'n, R. Chavarriaga, P. W. Ferrez, E. Lew, and J. R. Milla'n, The use of braincomputer interfacing in ambient intelligence, Constr. Ambient Intell., vol. 11, no. 6, pp. 268-285, 2008 .

10. M. Satyanarayanan, Pervasive computing: Vision and challenges, IEEE Pers. Commun., vol. 8, no. 4, pp. 10-17, Aug. 2001.

11. L. Ja"ncke, N. Shah, and M. Peters, Cortical activations in primary and secondary motor areas for complex bimanual movements in professional pianists, Cogn. Brain Res., vol. 10, no. 1-2, pp. 177-183, 2000.

12. L. D. Loukopoulos, K. Dismukes, and I. Barshi, The Multitasking Myth: Handling Complexity in Real-World Operations. Surrey, U.K.: Ashgate Pub. Co., 2009.

13. J. S. Kelso, D. L. Southard, and D. Goodman, On the coordination of two-handed movements, J. Exp. Psychol., Human Percep. Performance, vol. 5, no. 2, pp. 229-238, 1979.

14. C. T. Lin, L. W. Ko, M. H. Chang, J. R. Duann, J. Y. Chen, T. P. Su, and T. P. Jung, Review of wireless and wearable electroencephalogram systems and brain-computer interfaces VA minireview, Gerontology, vol. 56, no. 1, pp. 112-119, 2010.

15. C. B. Holroyd and M. G. H. Coles, The neural basis of human error processing: Reinforcement learning, dopamine, and the error-related negativity, Psychol. Rev., vol. 109, no. 4, pp. 679709, 2002. [102] M. Wexler and F. Klam, Movement prediction and movement production, J. Exp. Psychol., Human Percep. Performance, vol. 27, no. 1, pp. 48-64, 2001. 
16. N. Lee, A. J. Broderick, and L. Chamberlain, What is Fneuromarketing_? A discussion and agenda for future research, Int. J. Psychophysiol., vol. 63, no. 2, pp. 199-204, 2007.

17. T. O. Zander and S. Jatzev, Detecting affective covert user states with passive brain-computer interfaces, in Proc. 3rd Int. Conf. Affective Comput. Intell. Interaction (ACII) Workshops, 2009, DOI: 10.1109/ACII. 2009.5349456.

18. R. W. Picard, S. Papert, W. Bender, B. Blumberg, C. Breazeal, D. Cavallo, T. Machover, M. Resnick, D. Roy, and C. Strohecker, Affective learningVA manifesto, BT Technol. J., vol. 22, no. 4, pp. 253-269, 2004.

19. T. O. Zander, M. Gaertner, C. Kothe, and R. Vilimek, Combining eye gaze input with a braincomputer interface for touchless human-computer interaction, Int. J. Human-Comput. Interaction, vol. 27, no. 1 , pp. 38-51, 2010.

20. S. P. Kelly, E. C. Lalor, C. Finucane, G. McDarby, and R. B. Reilly, Visual spatial attention control in an independent brain-computer interface, IEE Trans. Biomed. Eng., vol. 52, no. 9, pp. 15881596, Sep. 2005.

21. K. R. Muller, M. Tangermann, G. Dornhege, M. Krauledat, G. Curio, and B. Blankertz, Machine learning for real-time single-trial EEG analysis: From brain-computer interfacing to mental state monitoring, J. Neurosci. Methods, vol. 167, no. 1, pp. 82-90, 2008.

22. A. Kim and L. Osterhout, The independence of combinatory semantic processing: Evidence from event-related potentials, J. Memory Lang., vol. 52, no. 2, pp. 205-225, 2005.

23. W. Liu and S. F. Chang, Robust multi-class transductive learning with graphs, in Proc. IEEE Conf. Comput. Vis. Pattern Recognit., 2009, pp. 381-388.

24. H. Heinrich, H. Gevensleben, and U. Strehl, Annotation: Neurofeedback-train your brain to train behaviour, J. Child Psychol. Psychiatry, vol. 48, no. 1, pp. 3-16, 2007.

25. M. Bilalic, R. Langner, R. Ulrich, and W. Grodd, Many faces of expertise: Fusiform face area in chess experts and novices, J. Neurosci., vol. 31, no. 28, pp. 10 206-10 214, 2011.

26. K. Yarrow, P. Brown, and J. W. Krakauer, Inside the brain of an elite athlete: The neural processes that support high achievement in sports,
Nature Rev. Neurosci., vol. 10, no. 8, pp. 585-596, 2009.

27. D. Linden and T. Lancaster, Functional Magnetic Resonance Imaging (FMRI)-based neurofeedback as a new treatment tool for depression, Eur. Psychiatry, vol. 26, no. 1, p. 937 , 2011, DOI: 10.1016/S0024-9338(11)72642-6.

28. H. Gevensleben, B. Holl, B. Albrecht, C. Vogel, D. Schlamp, O. Kratz, P. Studer, A. Rothenberger, G. Moll, and H. Heinrich, Is neurofeedback an efficacious treatment for ADHD? A randomised controlled clinical trial, J. Child Psychol. Psychiatry, vol. 50, no. 7, pp. 780-789, 2009.

29. R. P. Reddy, J. Rajan, I. Bagavathula, and T. Kandavel, Neurofeedback training to enhance learning and memory in patient with traumatic brain injury: A single case study, Int. J. Psychosocial Rehabil., vol. 14, no. 1, pp. 21-28, 2009.

30. K. B. Cannon, L. Sherlin, and R. R. Lyle, Neurofeedback efficacy in the treatment of a 43year-old female stroke victim: A case study, J. Neurotherapy, vol. 14, pp. 107-121, May 2010.

31. M. H. Berman and J. A. Frederick, Efficacy of neurofeedback for executive and memory function in dementia, Alzheimer's and Dementia, vol. 5, p. e8, Jul. 2009.

32. S. F. Cogan, Neural stimulation and recording electrodes, Annu. Rev. Biomed. Eng., vol. 10, pp. 275-309, 2008.

33. F. Fregni and A. Pascual-Leone, Technology insight: Noninvasive brain stimulation in neurologyVPerspectives on the therapeutic potential of rTMS and tDCS, Nature Rev. Neurol., vol. 3, no. 7, pp. 383-393, 2007.

34. W. J. Tyler, Y. Tufail, M. Finsterwald, M. L. Tauchmann, E. J. Olson, and C. Majestic, Remote excitation of neuronal circuits using low-intensity, low-frequency ultrasound, PLoS One, vol. 3, no. 10, p. e3511, 2008, DOI: 10.1371/journal.pone. 0003511.

35. J. Wells, C. Kao, E. D. Jansen, P. Konrad, and A. Mahadevan-Jansen, Application of infrared light for in vivo neural stimulation, J. Biomed. Opt., vol. 10, 064003, 2005.

36. T. O. Zander, C. Kothe, S. Jatzev, and M. Gaertner, Enhancing human-computer interaction with input from active and passive brain-computer interfaces, in Brain-Computer Interfaces. London, U.K.: Springer-Verlag, 2010, pp. 181-199. 


\section{www.rspsciencehub.com}

37. L. George and A. Le'cuyer, An overview of research on passive_ brain-computer interfaces for implicit human-computer interaction, in Proc. Int. Conf. Appl. Bionics Biomech. ICABBVWorkshop W1 Brain Computer Interfacing and Virtual Reality, 2010.

38. S. K. Lal and A. Craig, Driver fatigue: Electroencephalography and psychological assessment, Psychophysiology, vol. 39, no. 3, pp. 313-321, 2002.

39. J. R. Anderson, C. F. Boyle, and B. J. Reiser, "Intelligent tutoring systems", Science, vol. 228, no. 4698, pp. 456-462, 1985.

40. M. A. Carskadon and W. C. Dement, "Normal human sleep: An overview", in Principles and Practice of Sleep Medicine. St. Louis, MO: Elsevier Saunders, 2000, pp. 15-25.

41. Leuthardt EC, Schalk G, Roland J, Rouse A, Moran DW. Evolution of brain-computer interfaces: going beyond classic motor physiology. Neurosurg Focus Jul;2009 27(1):E4. [PubMed: 19569892].

42. Murase N, Duque J, Mazzocchio R, Cohen LG. Influence of interhemispheric interactions on motor function in chronic stroke. Ann Neurol Mar;2004 55(3):400-9. [PubMed: 14991818].

43. Daly JJ, Yin F, Perepezko EM, Siemionow V, Yue GH. Prolonged cognitive planning time, elevated cognitive effort, and relationship to coordination and motor control following

stroke. Neural Systems and Rehabilitation Engineering, IEEE Transactions on 2006;14(2):168-171.

44. Enzinger C, Ropele S, Fazekas F, Loitfelder M, Gorani F, Seifert T, Reiter G, Neuper C, Pfurtscheller G, Muller-Putz G. Brain motor system function in a patient with complete spinal cord injury following extensive brain-computer interface training. Exp Brain Res Sep;2008 190(2):215-23. [PubMed: 18592230]

45. Daly JJ, Hogan N, Perepezko EM, Krebs HI, Rogers JM, Goyal KS, Dohring ME, Fredrickson E, Nethery J, Ruff RL. Response to upper-limb robotics and functional neuromuscular stimulation following stroke. J Rehabil Res Dev Nov-Dec;2005 42(6):723-36. [PubMed: 16680610]46. Daly, JJ.; Cheng, RC.; Hrovat, K.; Litinas, KH.; Rogers, JM.; Dohring, ME. Development and testing of noninvasive BCI + FES/robot system for use in motor

\section{Volume 02 Issue 06 June 2020}

re-learning after stroke. Proc International Functional Electrical Stimulation Society; 2008. 